\title{
Impacto de la simbiosis micorrítica arbuscular en el crecimiento temprano del cultivo de tara (Caesalpinia spinosa (Molina) Kuntze)
}

\author{
Impact of the arbuscular mycorrhizal symbiosis on the early growth of tara \\ (Caesalpinia spinosa (Molina) Kuntze)
}

\author{
Sheena Sangay-Tucto ${ }^{1,2}$, Hervé Sanguin ${ }^{3}$, Estelle Tournier ${ }^{3}$, Jean Thioulouse ${ }^{4}$, Yves Prin $^{3}$ \\ y Robin Duponnois ${ }^{2}$
}

\begin{abstract}
Resumen
Tara (Caesalpinia spinosa) ha sido cultivada durante muchos años en bosques naturales, principalmente para la recolección de sus vainas y sus semillas dado su alto valor por sus múltiples usos desde tiempos antiguos. Sin embargo, poco se conoce sobre la ecología y el estado de conservación de los bosques de tara. La simbiosis micorrítica puede desempeñar un rol central en el crecimiento temprano de esta leguminosa y constituye un componente clave para la mejora de las prácticas de manejo. El objetivo de este estudio fue describir el estado micorrítico de tara en plantaciones localizadas en Perú y evaluar el impacto micorrítico sobre el crecimiento temprano de plántulas de Tara en condiciones de invernadero. Los resultados mostraron que la tara se asoció principalmente con hongos micorríticos arbusculares de la familia Glomeraceae, notablemente Rhizophagus spp. La micorrización controlada con esporas de $R$. irregularis mejoró significativamente el crecimiento de tara en invernadero, así como la absorción de nutrientes como el fósforo (P) y el nitrógeno $(\mathrm{N})$. Por lo tanto, C. spinosa podría ser considerada como "altamente dependiente de la micorriza". Estos resultados destacan la necesidad de considerar la simbiosis micorrítica arbuscular para mantener en forma sostenible la productividad y estabilidad de las plantaciones de tara.
\end{abstract}

Palabras clave: Caesalpinia spinosa; simbiosis arbuscular micorrítica; diversidad; dependencia micorrítica.

\footnotetext{
${ }^{1}$ Facultad de Ciencias y Filosofía, Unidad de Biomineria y Medioambiente, Universidad Peruana Cayetano Heredia, Perú. E-mail: sheenasangay@gmail.com

${ }^{2}$ Laboratoire des Symbioses Tropicales et Méditerranéennes-UMR 113, Instituto de Investigación para el Desarrollo (IRD), Francia.

${ }^{3}$ Laboratoire des Symbioses Tropicales et Méditerranéennes-UMR 113, Centro de Cooperación Internacional en Investigación Agronómica para el Desarrollo (CIRAD), Francia.

${ }^{4}$ Laboratoire de Biométrie et Biologie Évolutive, Universidad de Lyon, Francia.
} 


\begin{abstract}
Tara (Caesalpinia spinosa) has been cultivated for many years by managing natural forests, mainly for pod and seed extraction, and is highly appreciated for its multiple uses since ancient times. However little is known about the ecology and conservation status of tara forests. Mycorrhizal symbiosis may play a central role in the early growth of this legume tree and constitute a key component for the improvement of management practices. The aim of this study was to describe the tara mycorrhizal status in plantations located in Peru and to evaluate the mycorrhizal impacts on the early growth of tara seedlings in nursery conditions. The results showed that the tara was mainly associated with AM fungi affiliated to the Glomeraceae family, notably Rhizophagus spp. The controlled mycorrhization with spores of Rhizophagus irregularis significantly enhanced the tara growth in glasshouse, as well as the $\mathrm{P}$ and $\mathrm{N}$ nutrient uptake. Hence $C$. spinosa could be considered as "highly dependent on mycorrhiza". These results emphasize the need to consider the AM symbiosis in order to sustainably maintain the productivity and stability of tara plantations.
\end{abstract}

Key words: Caesalpinia spinosa; AM symbiosis; diversity; mycorrhizal dependency.

\section{Introducción}

Los bosques secos son los ecosistemas tropicales más debilitados que resultan de las altas presiones antropogénicas. Se ha reportado que el $66 \%$ del ecosistema de bosque tropical seco ha sido asignado a otros usos de la tierra, particularmente en Perú donde el porcentaje de pérdida ha alcanzado el 95\% (Portillo-Quintero y Sánchez-Azofeifa 2010). Factores antropogénicos, como prácticas culturales insostenibles o sobreexplotación de especies con alto valor comercial, han llevado a problemas de regeneración que afectan negativamente la sostenibilidad del bosque (Varghese et al. 2015). Por ejemplo, Balaguer et al. (2011), reportaron que un bosque de Caesalpinia spinosa (Molina) Kuntze (tara) fue sometido a bajos niveles de regeneración en Atiquipa (Perú). Más recientemente, se ha informado de que en la mayoría de las plantaciones de tara evaluadas en diferentes localidades del Perú se detectó un proceso de regeneración limitado como resultado de la excesiva recolección de semillas o pastoreo (Cordero et al. 2016). La tara se ha cultivado durante muchos años a través del manejo de bosques naturales, principalmente para la recolección de vainas y semillas (Larrea 2011). Aunque esta especie arbórea es altamente apreciada por sus múltiples usos desde la antigüedad, poco se sabe sobre la ecología y el estado de conservación de los bosques de tara, limitando el desarrollo de prácticas de manejo sostenible (Larrea 2011). La estructura de la población y los patrones espaciales de las cubiertas forestales se han considerado ampliamente para evaluar su dinámica de regeneración (Niklas et al. 2003). Por lo general, estos criterios resultan del efecto y la interacción entre los factores ambientales, las características de las especies arbóreas, las intra e interacciones específicas entre planta y planta, las interacciones entre planta y animal y el impacto antropogénico en el tiempo (Wiegand y Moloney 2004). Numerosos estudios también han indicado que las interacciones micorríticas pueden impulsar las funciones de los ecosistemas y éxito del establecimiento de las plántulas, uno de los procesos más críticos que determinan la sostenibilidad de los ecosistemas (Dickie et al. 2002; van der Heijden et al. 2006; Ibanez et al. 2015). Aunque algunos estudios han reportado que Caesalpinioideae presenta una gran variación de colonización radicular con hongos micorríticos arbusculares (AM) (Frioni et al. 1999), no existe información sobre el estado micorrítico de C. spinosa y su dependencia micorrítica. Los objetivos en la presente investigación fueron: (i) determinar el estado 
micorrítico de tara en plantaciones ubicadas en Perú y (ii) evaluar los impactos micorríticos sobre el crecimiento temprano de plántulas de tara en condiciones de invernadero. Se plantea la hipótesis de que esta especie arbórea se asocia en condiciones de campo con hongos AM y que el manejo de la infectividad micorrítica del suelo podría incrementar de forma sostenible la productividad y estabilidad de las plantaciones de tara en el Perú.

\section{Materiales y Métodos}

\section{Materiales}

\section{Material vegetal}

- Raíces de árboles de tara C. spinosa (Molina) Kuntze

- Semillas de tara C. spinosa (Molina) Kuntze

\section{Métodos}

\section{Lugar de ejecución}

La parte experimental de la presente investigación se realizó en el Laboratorio de Simbiosis Tropicales y Mediterráneas (LSTM), en el campus internacional de Baillarguet en Montferrier sur lez en la ciudad de Montpellier en Francia.

\section{Toma de muestra}

La recolección de muestras de raíces (10 $\mathrm{cm}$ de profundidad) se llevó a cabo en una plantación de tara en Canchacaya, de 2 zonas diferentes que denominamos $\mathrm{H} 1$ y $\mathrm{H} 2$ para el presente estudio. Ubicadas en la región de Huánuco (S $10^{\circ} 10.654^{\prime}$; W $076^{\circ} 10.129^{\prime}$ ), provincia de Ambo a 2459 msnm. Por cada zona, se seleccionaron cinco árboles al azar, y por cada árbol se recolectaron 3 muestras de raíces.

Las muestras de raíces fueron colocadas en bolsas de plástico de primer uso las que se encontraban debidamente rotuladas y luego transportadas en una caja frigorífica portátil que mantuvieran la temperatura a $4^{\circ} \mathrm{C}$ aproximadamente, hasta que fueran procesadas en el laboratorio.

En el laboratorio, las raíces fueron lavadas cuidadosamente con agua de caño y se ob- servaron bajo un estereomicroscopio (Olympus SZ H10 research stereomicroscope) con aumentos de $4 \mathrm{x}$ para detectar la presencia de nódulos rizobianos y/o ectomicorrizas, luego se secaron para análisis molecular.

\section{Evaluación de la estructura de la comunidad AM}

\section{- Extracción de ADN}

Se trituraron las raíces secas en nitrógeno líquido para homogenización y se extrajo el ADN en una submuestra (40 mg) usando un homogeneizador FastPrep-24 (MP biomedicals Europe, Illkirch, France) y el kit FastDNA ${ }^{\circledR}$ SPIN (MP biomedicals Europe), de acuerdo con las instrucciones del fabricante. Se mejoró la calidad de los extractos de ADN, añadiendo 20-30 mg de polivinilpolipirrolidona (PVPP) durante la primera etapa de extracción de ADN. Se realizaron tres repeticiones por muestra de raíz. Se comprobó la integridad del ADN en gel de agarosa al 1,5\% y se realizó la cuantificación del ADN utilizando el kit de ensayo Quant-iT ${ }^{\mathrm{m} m}$ PicoGreen $^{\circledR}$ dsDNA (Invitrogen, St. Aubin, Francia).

\section{- Análisis molecular de la diversidad de hongos micorríticos arbusculares a través de secuencia- miento (tecnología Illumina)}

Las muestras de ADN extraídas a partir de la raíz de árboles de tara, fueron purificadas para amplificar el gen $18 \mathrm{~S}$ ARNr, utilizando cebadores específicos NS31 y AML2 (Simon et al. 1992; Lee et al. 2008) con la finalidad de evaluar la diversidad de los hongos AM (Glomeromycota). Los amplicones obtenidos fueron secuenciados por medio de una cadena de secuenciamiento obtenidos a través de la tecnología Miseq illumina (1x300 pb) (Genotoul, Toulouse, Francia). Las reacciones de PCR para la amplificación fueron realizadas en dos etapas: i) la primera etapa se llevó a cabo en un volumen final de $50 \mu \mathrm{L}$ usando cebadores modificados NS31 (5'-ctttccctacacgacgctcttccgatctTTGGAGGGCAAGTCTGGTGCC-3') y AML2 (5’-ggagttcagacgtgtgctcttccgatctGAACCCAAACACTTTGGTTTCC-3') 0,6 $\mu \mathrm{M}$ de cada uno respectivamente, $2 \mu \mathrm{L}$ de $\mathrm{ADN}$ (2 repeticiones de $\mathrm{ADN}$ extraído por 
muestra), $200 \mu \mathrm{M}$ de cada dNTP, $200 \mathrm{ng} / \mathrm{mL}$ BSA, GoTaq ${ }^{\circledR}$ ADN Polimerasa (2 unidades) y 1X Green GoTaq ${ }^{\circledR}$, Buffer de reacción (Promega, Charbonnieres, Francia). Las condiciones de PCR fueron las siguientes: $94^{\circ} \mathrm{C}$ por $5 \mathrm{~min}$; 30 ciclos de $94^{\circ} \mathrm{C}$ por 30 s, $58^{\circ} \mathrm{C}$ por $1 \mathrm{~min}, 72^{\circ} \mathrm{C}$ por 80s; una etapa de elongación final a $72^{\circ} \mathrm{C}$ por $5 \mathrm{~min}$. Se agruparon tres repeticiones de PCR por cada muestra, los cuales fueron purificados utilizando GFX PCR ADN y el kit de purificación de bandas a partir del gel (GE Healthcare Life Sciences, Vlizy-Villacoublay, Francia) siguiendo las indicaciones del fabricante; para la segunda etapa, se enviaron los productos de PCR a secuenciar a la plataforma Genotoul (Toulouse, Francia), a través de la tecnología Illumina.

\section{- Procesamiento de secuencias generadas por el secuenciamiento con la tecnología Illumina.}

Las secuencias de una hebra (NS31) obtenidas con el secuenciamiento por la tecnología Illumina fueron analizadas y se verificó su calidad usando el software Mothur de acuerdo con el procedimiento operativo estándar (http:// www.mothur.org/wiki/MiSeq_SOP) propuesto en Kozich et al. (2013), sin embargo, la calidad de las secuencias ubicadas en los extremos de los fragmentos de ADN obtenidos fue verificada usando la configuración Q30 (comando trim.seqs). A continuación, todas las secuencias fueron agotadas con códigos de barras y cebadores, y se eliminaron todas las secuencias $<150 \mathrm{pb}$ (bases ambigüas u homopolímeros superiores a $8 \mathrm{pb}$ ). Enseguida, se realizó un paso de pre-agrupamiento (Huse et al. 2010) para eliminar secuencias erróneas formadas probablemente por errores del secuenciamiento, también se eliminó quimeras basándose en el algoritmo Uchime (Edgar et al. 2011). Por último, se clasificaron las secuencias alineándolas con una base de datos basada en Glomeromycota de Krüger et al. (2012) y el método clasificador RDP-naïve implementado en Mothur (comando classify.seq). Todas las secuencias no-Glomeromycota ( $<40 \%$ de confianza limite a nivel familiar) fueron eliminadas. Se realizó el agrupamiento de secuencias en unidades taxonómicas operacionales (OTUs) utilizando dist.seqs y comandos de agrupamiento en Mothur, los OTUs solitarios fueron eliminados. Se normalizaron el número de secuencias entre cada muestra con el comando sub.sample $(19,114$ secuencias por muestra). El paso de submuestreo permitió reducir el número de falsos OTUs, lo cual es crucial para obtener una comparación confiable entre las muestras (Gihring et al. 2012) Finalmente, los OTUs definidos con una divergencia del 3\% (97\% de similitud) fueron seleccionados para la afiliación taxonómica usando el comando classify.otu.

\section{Ensayos a nivel de invernadero}

\section{- Desinfección y germinación de semillas de C. spinosa}

Las semillas de C. spinosa, recogidas de la misma plantación de tara, se esterilizaron superficialmente con ácido sulfúrico concentrado $\left(\mathrm{H}_{2} \mathrm{SO}_{4}\right)$ al 95\% (Sanon 2009) durante $15 \mathrm{~min}$. Luego, se retiró la solución ácida y se enjuagaron las semillas durante $12 \mathrm{~h}$ cuatro veces en agua destilada estéril. Posteriormente, se transfirieron asépticamente en placas petri llenas con agar al $1 \%(\mathrm{w} / \mathrm{v})$ y germinaron en la oscuridad a $25^{\circ} \mathrm{C}$ durante 1 semana. Las semillas germinadas se utilizaron cuando las raíces tenían 1-2 cm de largo, las cuales se cultivaron en macetas de $250 \mathrm{~cm}^{3}$ llenas con una mezcla de tierra/arena $(1 / 4 ; \mathrm{v} / \mathrm{v})$.

\section{- Inoculación del hongo}

Se produjo el inóculo fúngico $R$. irregularis en condiciones axénicas según Bécard y Fortin (1988). Considerando que $1 \mathrm{~kg}$ de este inóculo contiene $10^{6}$ esporas, se realizó un cálculo simple para obtener la cantidad necesaria según el tratamiento. Se procedió a inocular a razón de 0, 125, 250 y 500 esporas por maceta. Luego, estas se dispusieron en un diseño de bloques completos al azar con 10 repeticiones por tratamiento. Las plantas se mantuvieron con luz natural (duración del día aproximadamente: $10 \mathrm{~h}$, temperatura media de $22^{\circ} \mathrm{C}$, temperatura máxima (de día) de $25^{\circ} \mathrm{C}$ y mínima (de noche) de $15^{\circ} \mathrm{C}$ ). Estas fueron regadas diariamente con agua osmótica sin aporte de fertilizantes. 


\section{- Tratamiento de plantas post-cosecha}

Las plántulas de tara se cosecharon después de 4 meses de cultivo y sus raíces se lavaron con agua de caño. Se llevaron cinco plántulas de $C$. spinosa al horno ( 1 semana a $65^{\circ} \mathrm{C}$ ) para determinar el peso seco de la parte aérea. Se realizó la determinación de nitrógeno y fósforo a partir de tejidos vegetales tal y como se describe en Boudiaf et al. (2013). Se evaluó la colonización de AM como se describe en Ouahmane et al. (2006) utilizando la tinción con azul de tripano y la dependencia micorrítica de C. spinosa se expresó como el porcentaje de la diferencia entre la biomasa total de una planta micorrízada y la biomasa total de una planta no micorrizada dividido entre la biomasa total de la planta micorrizada (Plenchette et al. 1983). Se evaluó la longitud radicular de las plantas restantes de acuerdo con van der Heijden et al. (1998). Finalmente, las raíces fueron llevadas al horno (por 1 semana a $65^{\circ} \mathrm{C}$ ) para su secado y luego se pesaron.

\section{Análisis estadísticos}

Se trataron todos los datos con un análisis de varianza unidireccional (ANOVA). Las medias se compararon mediante la prueba de Newman-Keul's $(\mathrm{P}<0,05)$.

\section{Resultados}

\section{Presencia de nódulos rizobianos y/o ectomi- corrizas}

Los árboles muestreados de tara de 5 años de la plantación de Canchacaya en Huánuco, de ambas zonas, $\mathrm{H} 1$ y $\mathrm{H} 2$, no presentaron ectomicorrizas ni nódulos rizobianos en las raíces de tara recolectadas, lo que indica un estado simbiótico específico AM en tara.

\section{Evaluación de la estructura de la comunidad AM}

La diversidad de hongos AM registrada en raíces de tara mostró un predominio de la familia de Glomeraceae (98\% de secuencias), notablemente asociado a Rhizophagus, Sclerocystis y Glomeraceae incertae sedis (Figura 1).

\section{Tratamientos de plantas post-cosecha}

\section{- Parámetros de crecimiento}

El tratamiento que se resalta corresponde a la inoculación con 250 esporas que en comparación al control incrementa en 1,8 para el peso seco de la parte radicular, en 2,6 para la parte aérea, en 2,2 para la biomasa total, en 2,1 para la longitud radicular (Cuadro 1).

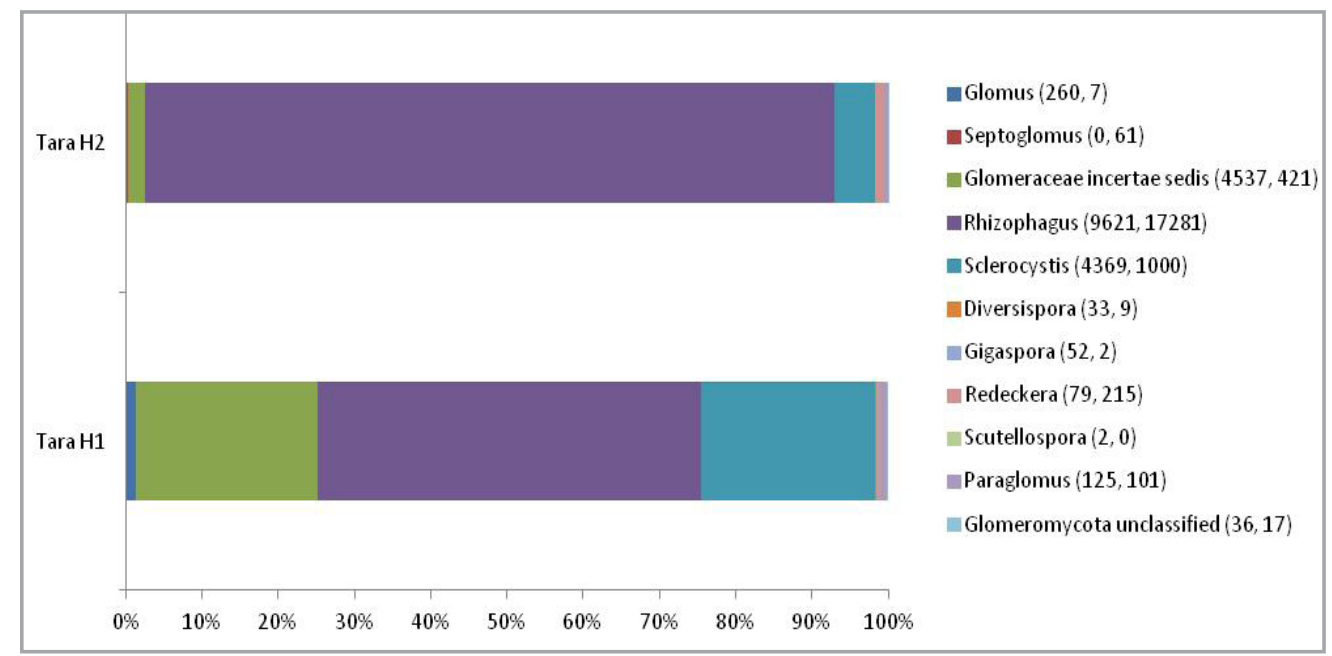

Figura 1. Estructura taxonómica de las comunidades de hongos AM asociados con los árboles de C. spinosa (tara) (plantaciones $\mathrm{H} 1$ y H2). Los datos se normalizaron a 19114 secuencias por muestra. Los datos se expresan como abundancia relativa de cada grupo taxonómico (\% del número total de secuencias) para cada árbol. Los números entre paréntesis indican el número de secuencias para $\mathrm{H} 1 \mathrm{y} \mathrm{H} 2$ respectivamente. 


\begin{tabular}{|c|c|c|c|c|}
\hline & \multicolumn{4}{|c|}{ Número de esporas AM inoculadas por maceta } \\
\hline & Control (0) & 125 & 250 & 500 \\
\hline Biomasa aérea (mg peso seco) & $161,3(26,9)^{(1)} \mathrm{a}^{(2)}$ & $302,5(79,2) b$ & $417,9(55,7) b$ & $392,3(26,9) b$ \\
\hline $\begin{array}{c}\text { Biomasa radicular (mg peso } \\
\text { seco) }\end{array}$ & $184,1(23,8) \mathrm{a}$ & $380,2(95,6) b$ & $336,8(20,5) b$ & $324,1(90,1) b$ \\
\hline Biomasa Total (mg peso seco) & $345,4(25,9)$ a & $682,7(174,4)$ a & $754,7(69,9) b$ & $716,4(24,8) b$ \\
\hline Longitud radicular $(\mathrm{cm})$ & $259,1(11,5) \mathrm{a}$ & $438,6(59,4) b$ & $542,3(93,9)$ bc & $585,5(24,5) \mathrm{c}$ \\
\hline Dependencia micorrítica (\%) & - & $47,4(11,9)$ a & $54,1(4,1)$ a & $51,2(7,3)$ a \\
\hline $\begin{array}{l}\text { Contenido de } \mathrm{N} \text { en la parte } \\
\text { aérea }\left(\mathrm{mg} \mathrm{g}^{-1} \text { peso seco) }\right.\end{array}$ & $2,12(0,07) \mathrm{a}$ & $2,98(0,12) b$ & $3,04(0,09) b c$ & $3,12(0,02) \mathrm{c}$ \\
\hline $\begin{array}{l}\text { Contenido de } \mathrm{N}_{\text {en la parte }} \\
\text { radicular (mg g } \text { peso seco) }^{-1} \text { eso }\end{array}$ & $0,78(0,02)$ a & $0,89(0,03) \mathrm{b}$ & $0,91(0,04) b$ & $0,98(0,08) b$ \\
\hline $\begin{array}{l}\text { Contenido de } \mathrm{P} \text { en la parte } \\
\text { aérea }\left(\mathrm{mg} \mathrm{g}^{-1} \text { peso seco) }\right.\end{array}$ & $0,11(0,02) \mathrm{a}$ & $0,23(0,04) \mathrm{b}$ & $0,29(0,02) b c$ & $0,38(0,02) \mathrm{c}$ \\
\hline $\begin{array}{l}\text { Contenido de } \mathrm{P} \text { en la parte } \\
\text { radicular ( } \mathrm{mg} \mathrm{g}^{-1} \text { peso seco) }\end{array}$ & $0,23(0,02)$ a & $0,25(0,03) \mathrm{a}$ & $0,31(0,02) \mathrm{b}$ & $0,32(0,03) \mathrm{b}$ \\
\hline
\end{tabular}

Cuadro 1. Impacto de la inoculación de esporas de R. irregularis en el crecimiento y contenido de nutrientes de plántulas de C. spinosa después de 4 meses de cultivo en condiciones de invernadero. ${ }^{(1)}$ Error standard. ${ }^{(2)}$ Dato en la misma línea seguido por la misma letra no son significativamente diferente de acuerdo a la prueba Newman-Keul's $(\mathrm{P}<0,05)$.

\section{- Contenido de nutrientes (nitrógeno y fósforo)}

Todas las plantas inoculadas fueron significativamente mejores que el control sin inocular, a excepción del tratamiento inoculado con 125 esporas, cuyo contenido de fósforo (en la parte radicular) fue similar al control (Cuadro 1).

\section{- Dependencia micorrítica}

La dependencia micorrítica fue de aproximadamente al 50\% en C. spinosa.

\section{Discusión}

Las Glomeraceae son las más ampliamente distribuídas en los ecosistemas (Opik et al. 2010), con especies AM (por ejemplo, Funneliformis mosseae y $R$. irregularis), mostrando una distribución geográfica extremadamente grande, potencialmente resultante de una falta de especialización de las condiciones ambientales (Rosendahl et al. 2009). La comunidad de hongos micorríticos arbusculares asociados a la C. spinosa en el presente estudio, están de acuerdo con los registrados en árboles Ceratonia siliqua L., una leguminosa esclerófila pertene- ciente también a la subfamilia de Caesalpinaceae (Manaut et al. 2015). El predominio de Rhizophagus spp. (70\% de las secuencias) puede sugerir un rol funcional importante en relación con el cultivo de tara. En consecuencia, se utilizó una cepa de $R$. irregularis en experimentos de micorrización controlada para evaluar el efecto en el crecimiento y nutrición del cultivo. Numerosos estudios han demostrado el efecto benéfico de $R$. irregularis sobre el crecimiento de otras especies arbóreas en condiciones controladas (Duponnois y Plenchette 2003; Dabire et al. 2007; Kisa et al. 2007) y después de la siembra en campo (Duponnois et al. 2007; Bilgo et al. 2012). En el presente estudio, la inoculación con dicho hongo micorrítico aumentó significativamente el crecimiento de las plántulas de tara en comparación a plantas no inoculadas.

Dado que los resultados de dependencia micorrítica en C. spinosa fue de $50 \%$, esto indica que esta especie podría clasificarse como "micorrítica obligatoria" (Brundett 1991) o como "altamente dependiente de la micorriza" (Habte y Manjunath 1991). 


\section{Agradecimientos}

IRD, Embajada de Francia, Ministerio de Educación Nacional, Enseñanza Superior e Investigación de la República Francesa y Red Universitaria Raúl Porras Barrenechea/ CONCYTEC-FONDECYT, UPCH y UNALM.

\section{Bibliografía}

Balaguer, L; Arroyo-García, R; Jiménez, P; et al. 2011. Forest restoration in a fog oasis: evidence indicates need for cultural awareness in constructing the reference. PloS One 6:e23004 . doi: 10.1371/journal.pone.0023004

Bécard, G; Fortin, JA. 1988. Early events of vesicular arbuscular mycorrhiza formation on $\mathrm{Ri}$ T-DNA transformed roots. New Phytol, 108: 211-218.

Bilgo, A; Sangare, SK; Thioulouse, J; Prin, Y; Hien, V; Galiana, A; Baudoin, E; Hafidi, M; Bâ, MA; Duponnois, R. 2012. Response of native soil microbial functions to the controlled mycorrhization of an exotic tree legume, Acacia holosericea in a Sahelian ecosystem. Mycorrhiza 22: 175-187

Boudiaf, I; Baudoin, E; Sanguin, H; Beddiar, A; Thioulouse, J; Galiana, A; Prin, Y; Le Roux, C; Lebrun, M; Duponnois, R. 2013. The exotic legume tree species, Acacia mearnsii, alters microbial soil functionalities and the early development of a native tree species Quercus suber, in North Africa. Soil Biol Biochem 65: 172-179

Brundrett, MC. 1991. Mycorrhizas in natural ecosystems. In: Macfayden, A; Begon, $\mathrm{M}$ and Fitter, AH (eds), advances in Ecological Research, Vol. 21. Academic Press Ltd, London, p. 171-313.

Cordero, I; Jimenez, MD; Delgado, JA; Villegas, L; Balaguer, L. 2016. Spatial and demographic structure of tara stands (Caesalpinia spinosa) in Peru: Influence of present and past forest management. For. Ecol. Manage. 377: 71-82

Dabire, AP; Hien, V; Kisa, M; Bilgo, A; Sangare, KS; Plenchette, C; Galiana, A; Prin, Y; Duponnois, R. 2007. Responses of soil microbial catabolic diversity to arbuscular mycorrhizal inoculation and soil disinfection. Mycorrhiza 17: 537-545
Dickie, IA; Koide, RT; Steiner, KC. 2002. Influences of established trees on mycorrhizas, nutrition, and growth of Quercus rubra seedlings. Ecol Monogr 72: 505-521

Duponnois, R; Plenchette, C. 2003. A mycorrhiza helper bacterium (MHB) enhances ectomycorrhizal and endomycorrhizal symbiosis of Australian Acacia species. Mycorrhiza 13: 85-91

Duponnois, R; Plenchette, C; Prin, Y; Ducousso, M; Kisa, M; Bâ, AM; Galiana, A. 2007. Use of mycorrhizal inoculation to improve reafforestation process with Australian Acacia in Sahelian ecozones. Ecol engin 29: 105-112

Edgar, RC; Haas, BJ; Clemente, JC; Quince, C; Knight, R. 2011. UCHIME improves sensitivity and speed of chimera detection. Bioinformatics 27: 2194-2200

Frioni, L; Minasian, H; Volfovicz, R. 1999. Arbuscular mycorrhizae and ectomycorrhizae in native tree legumes in Uruguay. For Ecol Manage 115: 41-47

Gihring, TM; Green, SJ; Schadt, CW. 2012. Massively parallel rRNA gene sequencing exacerbates the potential for biased community diversity comparisons due to variable library sizes. Environ Microbiol 14: 285-290

Habte, M; Manjunath, A. 1991. Categories of vesicular-arbuscular mycorrhizal dependency of host species. Mycorrhiza, 1: 3-12

Huse, SM; Welch, DM; Morrison, HG; Sogin, ML. 2010. Ironing out the wrinkles in the rare biosphere through improved OTU clustering. Environ Microbiol 12: 1889-1898.

Ibanez, B; Gomez-Aparicio, L; Avila, JM; Perez-Ramos, IM; Garcia, LV; Maranon, T. 2015. Impact of tree decline on spatial patterns of seedling-mycorrhiza interactions: Implications for regeneration dynamics in Mediterranean forests. For Ecol Manage 353: 1-9

Kisa, M; Sanon, A; Thioulouse, J; Assigbetse, K; Sylla, S; Spichiger, R; Dieng, L; Berthelin, J; Prin, Y; Galiana, A; Lepage, M; Duponnois, R. 2007. Arbuscular mycorrhizal symbiosis can counterbalance the negative influence of the exotic tree species Eucalyptus camaldulensis on the structure and functioning of soil microbial 
communities in a sahelian soil. FEMS Microbiol Ecol 62: 32-44

Kozich, JJ; Westcott, SL; Baxter, NT; Highlander, SK; Schloss, PD. 2013. Development of a dual-index sequencing strategy and curation pipeline for analyzing amplicon sequence data on the MiSeq Illumina sequencing platform. Appl Environ Microbiol 79: 5112-20

Krüger, M; Krüger, C; Walker, C; Stockinger, H; Schüssler, A. 2012. Phylogenetic reference data for systematics and phylotaxonomy of arbuscular mycorrhizal fungi from phylum to species level. New phytol 193: 970-984.

Larrea, M. 2011. La tara, guarango o taya (Caesalpinia spinosa) en la región andina: criterios ambientales para la sustentabilidad de su aprovechamiento y manejo en Bolivia, Ecuador y Perú. ECOBONA, Serie de Capacitación No. 5. Programa Regional ECOBONA-INTERCOOPER, Quito, Peru.

Lee, J; Lee, S; Young, JPW. 2008. Improved PCR primers for the detection and identification of arbuscular mycorrhizal fungi. FEMS Microbiol Ecol 65: 339-349

Manaut, N; Sanguin, H; Ouahmane, L; Bressan, M; Thioulouse, J; Baudoin, E; Galiana, A; Hafidi, M; Prin, Y; Duponnois, R. 2015. Potentialities of ecological engineering strategy based on native arbuscular mycorrhizal community for improving afforestation programs with carob trees in degraded environment. Ecol Eng 79: 113-119

Niklas, KJ; Midgley, JJ; Rand, RH. 2003. Tree size frequency distributions, plant density, age and community disturbance. Ecol Lett 6: 405-411.

Öpik, M; Vanatoa, A; Vanatoa, E; Moora, M; Davison, J; Kalwij, JM; Reier, U; Zobel, M. 2010. The online database MaarjAM reveals global and ecosystemic distribution patterns in arbuscular mycorrhizal fungi (Glomeromycota). New Phytol 188: 223-241

Ouahmane, L; Hafidi, M; Plenchette, C; Kisa, M; Boumezzough, A; Thioulouse, J; Duponnois, R. 2006. Lavandula species as accompanying plants in Cupressus replanting strategies: effect on plant growth, mycorrhizal soil infectivity and soil microbial catabolic diversity. Appl Soil Ecol 34: 190-199
Plenchette, C; Fortin, JA; Furlan, V. 1983. Growth responses of several plant species to mycorrhizae in a soil of moderate P fertility: I. Mycorrhizal dependency under field conditions. Plant Soil 70: 199-209

Portillo-Quintero, CA; Sánchez-Azofeifa, GA. 2010. Extent and conservation of tropical dry forests in the Americas. Biol. Conserv. 143: 144-155

Rosendahl, S; McGee, P; Morton, JB. 2009. Lack of global population genetic differentiation in the arbuscular mycorrhizal fungus Glomus mosseae suggests a recent range expansion which may have coincided with the spread of agriculture. Mol Ecol 18: 4316-4329

Sanon, A. 2009. Le concept de niche écologique associé à la co-existence des espèces végétales : mise en évidence du rôle de la symbiose mycorhizienne et de sa microflore associée dans la structuration de la strate herbacée en milieu tropical.

van der Heijden, MGA; Klironomos, JN; Ursic, M; Moutoglis, P; Streitwolf-Engel, R; Boller, T; Wiemken, A; Sanders, IR. 1998. Mycorrhizal fungal diversity determines plant bio-diversity, ecosystem variability and productivity. Nature 396: 69-72

van der Heijden, MGA; Streitwolf-Engel, R; Riedl, R; et al. 2006. The mycorrhizal contribution to plant productivity, plant nutrition and soil structure in experimental grassland. New Phytol 172:739-752

Varghese, A; Ticktin, T; Mandle, L; Nath, S. 2015. Assessing the effects of multiple stressors on the recruitment of fruit harvested trees in a tropical dry forest, Western Ghats, India. PLoS One 10, e0119634.

Wiegand, T; Moloney, KA. 2004. Rings, circles, and null-models for point pattern analysis in ecology. Oikos 104: 209-229. 